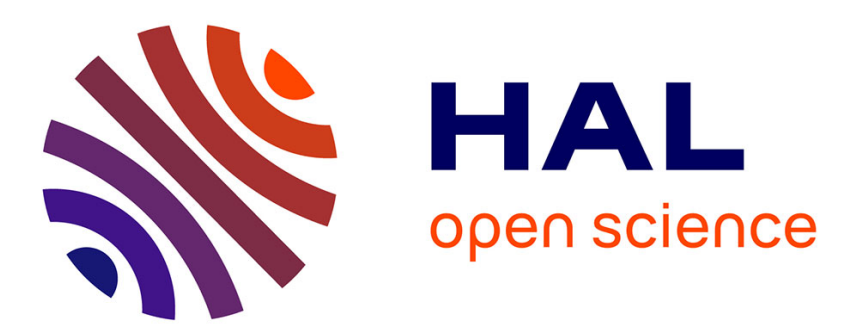

\title{
Multi-coefficient goal programming in thinning schedules to increase carbon sequestration and improve forest structure
}

Ying-Ta Chen, Ching-Ter Chang

\section{- To cite this version:}

Ying-Ta Chen, Ching-Ter Chang. Multi-coefficient goal programming in thinning schedules to increase carbon sequestration and improve forest structure. Annals of Forest Science, 2014, 71 (8), pp.907-915. $10.1007 / \mathrm{s} 13595-014-0387-\mathrm{z}$. hal-01102925

\section{HAL Id: hal-01102925 \\ https://hal.science/hal-01102925}

Submitted on 13 Jan 2015

HAL is a multi-disciplinary open access archive for the deposit and dissemination of scientific research documents, whether they are published or not. The documents may come from teaching and research institutions in France or abroad, or from public or private research centers.
L'archive ouverte pluridisciplinaire HAL, est destinée au dépôt et à la diffusion de documents scientifiques de niveau recherche, publiés ou non, émanant des établissements d'enseignement et de recherche français ou étrangers, des laboratoires publics ou privés. 


\title{
Multi-coefficient goal programming in thinning schedules to increase carbon sequestration and improve forest structure
}

\author{
Ying-Ta Chen $\cdot$ Ching-Ter Chang
}

Received: 22 January 2014 / Accepted: 15 May 2014 / Published online: 29 May 2014

(C) INRA and Springer-Verlag France 2014

\begin{abstract}
- Context Current decision analysis techniques are ineffective for planning thinning operation to improve the forest structure.

- Aims The purpose of this study is to use multi-coefficient goal programming (GP) to plan a thinning schedule that allows more carbon sequestration and diverse forest structure. - Methods A multi-coefficient GP is applied to plan a thinning schedule for a 2,633-ha plantation forest.

- Results This technique efficiently fine-tunes the thinning schedule to obtain 420,500 tons of carbon sequestration which was a little higher than the result by a multi-segment goal programming (MSGP). Moreover, a fixed-ratio multicoefficient GP is applied to efficiently generate mosaic of thinned areas with various thinning intensities. Although the captured carbon by a fixed-ratio multi-coefficient GP is lower, the thinned areas can provide various habitats for forest life with multiform contrasting edges.
\end{abstract}

Handling Editor: Barry Alan Gardiner

Contribution of the co-authors Ying-Ta Chen designed the experiment, wrote the paper, and run the data analysis. Ching-Ter Chang designed the experiment, wrote the paper, and supervised the work

Highlights Multi-coefficient goal programming (GP) can fine-tune the thinning schedules for increased carbon sequestration.

Fixed-ratio multi-coefficient GP can efficiently generate multiform edge effects and change forest structure.

These two techniques allow practicable planning of improved thinning alternatives.

Y.-T. Chen

Department of Tourism Management, Taiwan Shoufu University,

No.168, Nanshi Li, Madou Dist., Tainan, Taiwan, Republic of China

C.-T. Chang $(\square)$

Department and Graduate Institute of Information Management,

Chang Gung University, 259 Wen-Hwa 1st Road, Kwei-Shan,

Tao-Yuan 333, Taiwan, Republic of China

e-mail: chingter@mail.cgu.edu.tw
- Conclusion The use of a multi-coefficient GP allows practicable planning of better thinning alternatives to increase carbon sequestration and forest structure.

Keywords Thinning operation $\cdot$ Thinning schedule $\cdot$ Carbon sequestration · Biodiversity · Multi-coefficient goal programming $\cdot$ Fixed-ratio multi-coefficient goal programming

\section{Introduction}

Multi-criteria decision analysis (MCDA) is a decision-making approach that is often applied to solve forest management problems. It is a multi-attribute decision making (MADM) method and a multi-objective decision making (MODM) method that can be used to select discrete alternatives and solve multi-objective planning problems (Mendoza and Martins 2006). Heuristic methods of MADM that are used in forestry, such as ranking, analytic hierarchy process (AHP), group decision-making (GDM) techniques, and compromise programming and are often applied to landscape-scale planning, pulp and paper industries, forest management alternatives, watershed management alternatives, and forest management risks. Linear programming (LP), goal programming (GP), dynamic programming (DP), or integer programming (IP) are often used to solve most common forest MODM problems. Different types of LP and GP are used to allocate forest areas for multiple forest management, reforestation simulation, silvicultural treatment simulation, optimal rotation simulation, and spatial decision support systems for the environment. For thinning scheduling, risk and uncertainty evaluation, or harvest scheduling, DP is more appropriate. In contrast, IP allows the choice of the forest stand to be harvested, as well as options for road building and transportation. These MADM and MODM methods also can be integrated for 
various forest problems, such as the use of a combination of AHP with GP to allocate forestland according to forest managers' preferences (De-Steiguer et al. 2003; Mendoza and Martins 2006; Díaz-Balteiro and Romero 2008). For example, Diaz-Balteiro and Romero (2001) used AHP to obtain the preferential weights for a group of decision-makers and then incorporated these weights into a GP model for multiple forest management.

GP is an important operations research method that is generally used to solve MODM problems in forest management, such as the allocation of areas according to different prescriptions for multiple forest management. An alternative is here proposed that allocates limited resources to achieve multiple objectives as closely as possible (Charnes and Cooper 1961). However, a single goal with multiple choices is a common problem that cannot be solved by current GP methods. Chang (2007) firstly proposed multi-choice goal programming (MCGP) to solve this issue. Multi-segment goal programming (MSGP) was then developed to deal with the coefficients in a multi-choice situation within the constraints (Liao 2009). Multi-coefficient goal programming (multicoefficient GP) was proposed to deal with problems in which both the coefficients and the decision variables in the constraints are multi-choice situations (Chang et al. 2012). These new GP methods and their applications in forest management and other fields are introduced in this paper.

Figure 1 shows the decision process for choosing an appropriate GP method to solve various multiple forest management problems. When there are multi-objective forest planning problems, forest managers must decide whether the particular problem is a multi-choice problem. If this is a problem without a multi-choice set, forest managers can use traditional GP techniques, such as GP, lexicographic GP, weighted GP, min-max GP, integer GP, zero-one GP, fuzzy GP, interactive GP, and chance-constrained GP (Field 1973; Ignizio 1976; Lee 1972; Romero 1991; Tamiz et al. 1995). These GP methods allow forest managers to examine various alternatives for the forestland that achieve the goals set (e.g., timber production, visitors, wildlife, etc.) as fully as possible (Arp and Lavigne 1982; Bell 1976; Bottoms and Bartlett 1975; Díaz-Balteiro and Romero 1998, 2003; Hotvedt 1983; Oliveira et al. 2003).

When there are multiple-choice decision-making (MCDM) issues, forest managers require advanced GP methods to deal with multi-choice settings. Three multi-choice situations that are inherent in the goals or coefficients can be, respectively, solved by three new GP methods. In Fig. 1, the first multichoice situation is that forest managers would like to set one goal with multiple aspiration levels (e.g., the level of timber production required (high, medium, and low)). MCGP is a helpful technique for solving this problem. The use of MCGP in multiple forest management was proposed by Chen et al. (2011a), who proposed a 3-level MCGP to allow a more efficient search for better forest planning to achieve higher goals. MCGP is also used to help downstream companies to select the types of thin-film transistor liquid crystal displays (Lee et al. 2009), to construct supply chain models (Liao and Kao 2010), to choose suppliers in supply chains (Chang and $\mathrm{Ku}$ 2011; Liao and Kao 2011), to design quality management systems (Mahmoud et al. 2011), to help homebuyers (Chang et al. 2011), and for inventory management in supply chains (Paksoy and Chang 2010).

The second situation is that forest managers would like to set multiple choices for the coefficients (e.g., the selection of an appropriate thinning intensity (heavy, medium, or low) for an area to be thinned). Multi-segment GP (MSGP), developed by Liao (2009), is suitable for this situation. It is a complex process that allows forest managers to simulate various levels of thinning intensity for thinned land. Using both the thinned area and the thinning intensity as decision variables, Chen et al. (2011b) combined MSGP with LP to decrease the simulation times and allow a better thinning schedule for higher carbon sequestration. This method has also been used in pricing strategies for new products (Liao 2011) and for supplier selection (Chang et al. 2013).

The third situation is that multiple choices sets in the coefficients and the modulation of decision variables are considered at the same time in this study. The multicoefficient GP proposed by Chang et al. (2012) is used to solve this common problem of how to improve the forest structure of fragile forestland by thinning. Figure 2 shows a comparison of a MSGP and a multi-coefficient GP for formula setting and their application in the real world. Figure 2(a1) shows the MSGP set in the formula that assigns the area to be thinned to one of the thinning intensities. In real-world forest management, an MSGP changes only one part of the forest structure in each period, as shown in Fig. 2(a2). However, a multi-coefficient GP allocates each level of thinning intensity to an individual thinned area, as shown in Fig. 2(b1), where $60,40,20$, and $0 \%$ of trees are removed in the A1, A2, A3, and $\mathrm{A} 4$ areas. As Fig. 2(b2) shows, forest managers use this to efficiently produce a mosaic of forest structure with various thinning intensities. In this paper, these mosaics are assumed to diversify the animals and plants habitats. Adjacent mosaics also create edges with adjacent stands of different sizes and ages (Zhang et al. 2011). Some studies have deliberately planned harvesting schedules that create edge contrasts by clear-cutting between adjacent stands (Bertomeu and Romero 2001, 2002; Zhang et al. 2011). By creating an edge with clear-cutting, the adjacent nearby thinned areas scheduled by a multi-coefficient GP also offers an alternative for forest managers to increase forest biodiversity. The advantages of an MSGP and a multi-coefficient GP in scheduling thinning are summarized in Table 4 in the Appendix. Each of them can reduce the simulation time needed to efficiently select an appropriate thinning intensity. As a result, multi- 
Fig. 1 The decision process of choosing an appropriate GP method

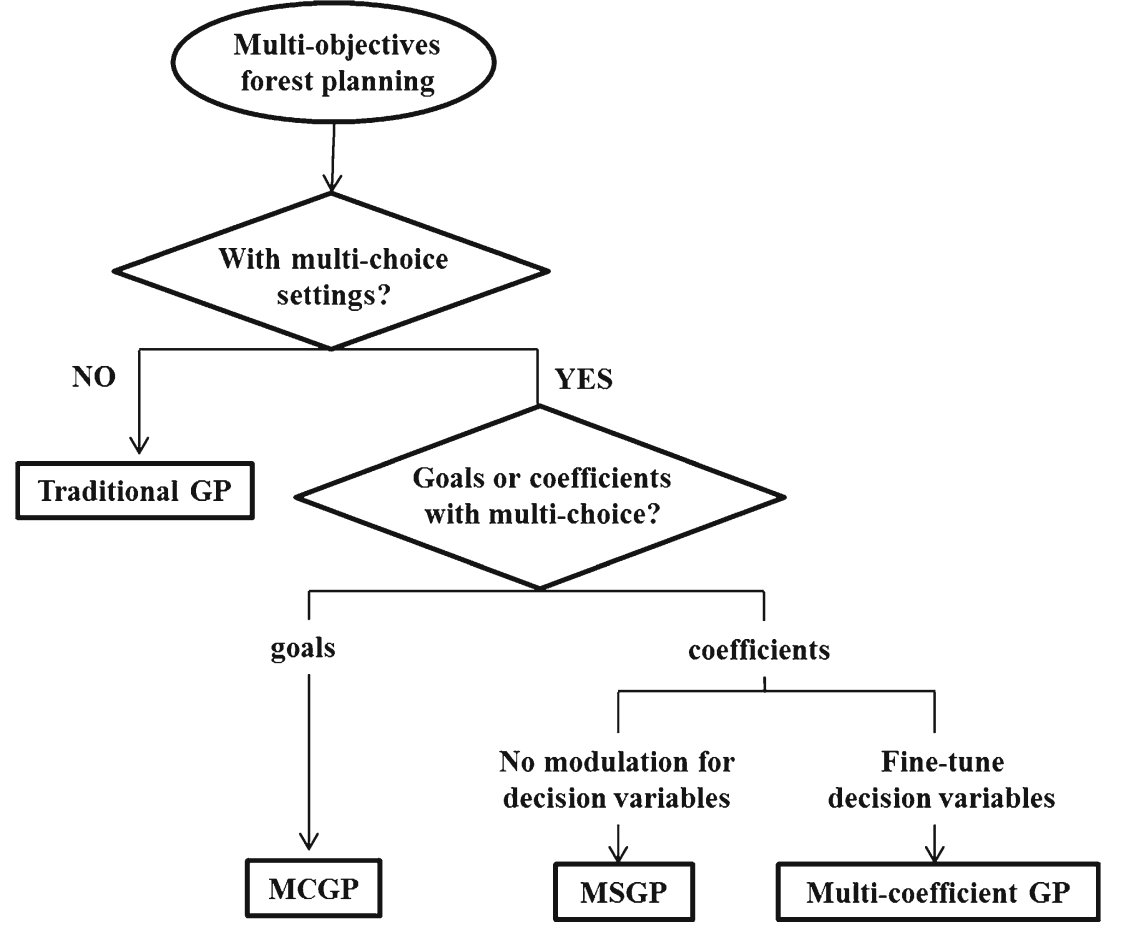

coefficient GP can fine-tune the area allocation. In addition, it is more efficient than MSGP in certain ways, such as controlling the composition of thinned areas with various thinning intensities in a forest or in assigning a thinned area for each thinning intensity.

According to the third forest resources and land use inventory compiled by the Taiwanese Forest Bureau in 1995, the average stock of forest is only $163.50 \mathrm{~m}^{3} / \mathrm{ha}$, which means that about 400,000 ha of plantation forest lack proper management and most are over-mature and infected by insects or diseases. Forests are not managed because of two restrictions: (a) traditional harvesting is harmful to fragile forestland and (b) the Taiwanese Forestry Law has limited the clear-cutting area to less than 5 ha. Therefore, thinning is the most appropriate practice for Taiwanese forests to improve tree health, timber production, and carbon sequestration ability.
Fig. 2 Concept of thinning schedule mapped by MSGP and multi-coefficient GP: $a 1$ and $b 1$, respectively, shows the formula setting of MSGP and multicoefficient GP. $a 2$ represents the monotonous forest structure created by MSGP, while $b 2$ refers to the biodiversity environment efficiently created by multi-coefficient GP

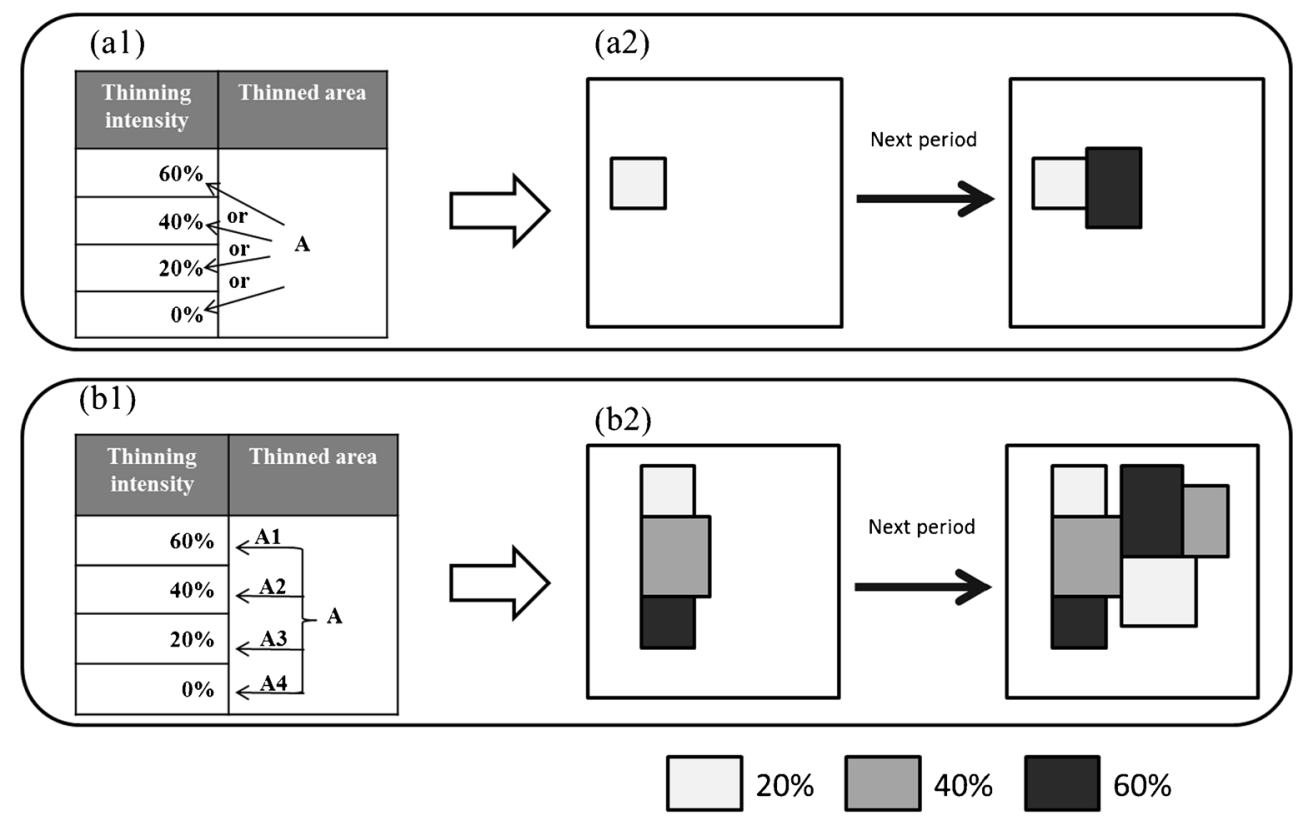

Formula concept

Application in real world 
Forest management in Taiwan currently focuses on the promotion of carbon sequestration and the health of plantation forests. To the best of our knowledge, multi-coefficient GP is the only OR method that deals with the third situation to finetune the thinned area, to quickly allocate thinned areas with various thinning intensities, to improve forest structure, and to create different levels of edge effect between adjacent thinned areas. This study uses a multi-coefficient GP to create a thinning schedule for two purposes: (a) to verify the advantages of this method in fine-tuning thinned areas to allow more carbon sequestration and (b) to use a fixed-ratio multi-coefficient GP to improve forest structure and to create a multiform edge effect that considers both carbon sequestration and biodiversity. This study firstly introduces the multi-coefficient GP and its applications in developing thinning schedules. The creation of a thinning schedule using a multi-coefficient GP and a fixedratio multi-coefficient GP is then described. Finally, the results of this study are verified against a real case.

\section{Material and methods}

\subsection{A planted forest case}

For the years 2008 to 2012, the harvested areas of the Hsinchu Forest District were, respectively, 27.60, 34.95, 50.69, 29.12, and 36.76 ha; $36.07,44.22,64.90,63.22$, and $74.67 \%$ of the total harvested areas of the Taiwanese Forest Bureau. In order to allow more efficient thinning, the Taiwanese Forest Bureau also selected this area in 2004 as an important thinning pattern district for demonstration purposes. If the thinning schedule from a multi-coefficient GP analysis is proven to stimulate the growth and health of forest stands in the Hsinchu Forest District, then this method can be applied to other forest districts in order to improve carbon sequestration and biodiversity in all ages of plantation forest in Taiwan.

A young Sugi (Cryptomeria japonica) stand in Hsinchu Forest District was chosen for demonstration because a good growth model exists for this species and thinning is often carried out in a young forest to help produce high quality timber. The area of this stand is 2,633 ha, and it contains $130.3 \mathrm{~m}^{3}$ timbers per hectare. The total volume of this forest is $343,000 \mathrm{~m}^{3}$. The 5 -year interval growth rates noted by Chen et al. (2011b), after different levels of thinning intensity, are displayed in Table 1. For example, the growth rate after $20 \%$ thinning is 0.4394 for the second period, 0.2754 for the third period, and 0.1834 for the fourth period.

2.2 Framework for a thinning schedule and its settings using multi-coefficient GP

Since 1992, the law has stated that a clear-cutting area must be no more than 5 ha, so the Taiwanese Forestry Bureau now faces the problem that most of the 400,000 ha of plantation forest are over-mature. In order to legally utilize the plantation forest, thinning is an important option that fulfills multiple forest management goals, such as stimulating the growth rate for more carbon sequestration, timber production, and forest health. Although an MSGP is helpful in planning a thinning schedule (Chen et al. 2011b), a multi-coefficient GP is a better method to plan a thinning schedule that provides more carbon sequestration and biodiversity in each of the eight Forest Districts in Taiwan. The original formula for a multicoefficient GP is expressed as follows.

$\min \mathrm{z}=\sum_{i=1}^{n}\left(d_{i}^{+}+d_{i}^{-}\right)$

Goals and constrants :

$\sum_{j=1}^{n} \sum_{k=1}^{m} C_{i k}(B) T_{i k} A_{i j}-d_{i}^{+}+d_{i}=G_{i}$
$\sum_{j=1}^{m} C_{i k}(B) A_{i j}=C_{i k}(B) R_{i j} A_{i}, i=1,2, \ldots, n$

A multi-coefficient GP and a traditional GP are calculated by the same principle to minimize the sum of the deviations between the target goal value and the achievement of $i$ th goals. The $G_{i}$ symbol refers to multiple forest management goals, such as timber production, visitors, and others. The positive and negative deviations $\left(d^{+}, d^{-}\right)$represent the distances which under- or over-achieve the actual goal values. The symbol, $T_{i k}$, denotes the $k$ th thinning intensity level (coefficients) that correspond to the $j$ th thinned area $\left(A_{i j}\right)$ of $A_{i}$. And $C_{i k}(B)$ is a function of the binary serial number that controls the combinations of $T_{i k}$ and $A_{i j}$. To clarify the features of a MSGP and a multi-coefficient GP, comparisons are listed in Table 5 in the Appendix. They both can set a coefficient to be multi-choice and then select one of the choices by a series of binary functions. But, a multi-coefficient GP is more effective in controlling the decision variables by a series of binary series functions that can decompose the decision variable $\left(A_{i}\right)$ into several parts $\left(A_{i j}\right)$ and control the ratios between them.

The usage of a multi-coefficient GP is demonstrated by the following five equations. Assuming that forest managers prefer to use heavy, medium, and low thinning intensity levels $\left(T_{i h}, T_{i m}\right.$, and $\left.T_{i l}\right)$, which involve the removal of 60,40 , and $20 \%$ of the trees, then the thinned area $\left(A_{i}\right)$ will be allocated for each thinning intensity level to be $A_{i 1}, A_{i 2}$, and $A_{i 3}$ using the binary variables, as Eqs. 1 and 2 show. The symbols $b_{1}$ and $b_{2}$ are binary variables so that their combination of $\left(b_{1}, b_{2}\right)=(1,1)$ or $(1,0)$ or $(0,1)$ controls the relationship between the thinned area $\left(A_{i j}\right)$ and the thinning intensity level $\left(T_{i k}\right)$ to be one of the three groups: $\left(0.6^{*} A_{i 1}, 0.2^{*} A_{i 2}, 0.4^{*} A_{i 3}\right)$, or $\left(0.4^{*} A_{i 1}, 0.6^{*} A_{i 2}, 0.2^{*} A_{i 3}\right)$, or $\left(0.2^{*} A_{i 1}, 0.4^{*} A_{i 2}, 0.6^{*} A_{i 3}\right)$. Notably, the meaning of $A_{i 1}, A_{i 2}$, and $A_{i 3}$ is decided by the ratio that is controlled by the binary variables. The $A_{i 2}$ symbol, for 
Table 1 The growth rate of 11-20 age-class after different thinning intensity in each period

\begin{tabular}{|c|c|c|c|c|c|c|c|c|c|c|}
\hline & \multicolumn{4}{|c|}{ Thinning during first period } & \multicolumn{3}{|c|}{ Thinning during second period } & \multicolumn{2}{|c|}{ Thinning during third period } & \multirow{2}{*}{$\begin{array}{l}\text { Thinning during fourth period } \\
4\end{array}$} \\
\hline & 1 & 2 & 3 & 4 & 2 & 3 & 4 & 3 & 4 & \\
\hline $0 \%$ & 0.4462 & 0.2916 & 0.2125 & 0.1607 & & & & & & \\
\hline $20 \%$ & 0.6115 & 0.3586 & 0.2361 & 0.1699 & 0.4393 & 0.2754 & 0.1834 & 0.3511 & 0.2209 & 0.2934 \\
\hline $40 \%$ & 1.2555 & 0.5897 & 0.3126 & 0.1987 & 0.6548 & 0.3613 & 0.2132 & 0.5534 & 0.3032 & 0.4871 \\
\hline $60 \%$ & 0.8528 & 0.4501 & 0.2673 & 0.1818 & 1.0145 & 0.4923 & 0.2566 & 0.8910 & 0.4286 & 0.8103 \\
\hline
\end{tabular}

example, represents the thinned area with low, heavy, and medium thinning intensity, respectively, in the first, second, and third group.

1. $\left[T_{i h} b_{1} b_{2}+T_{i m} b_{1}\left(1-b_{2}\right)+T_{i l} b_{2}\left(1-b_{1}\right)\right] A_{i 1}+\left[T_{i h} b_{1}\left(1-b_{2}\right)+\right.$ $\left.T_{i m} b_{2}\left(1-b_{1}\right)+T_{i l} b_{1} b_{2}\right] A_{i 2}+\left[T_{i h} b_{1}\left(1-b_{2}\right)+T_{i m} b_{2}\left(1-b_{1}\right)+\right.$ $\left.T_{i l} b_{1} b_{2}\right] A_{i 3}$

2. $A_{i}=A_{i 1}+A_{i 2}+A_{i 3}$

3. $A_{i 1}=\left[R_{i 1} b_{1} b_{2}+R_{i 2} b_{1}\left(1-b_{2}\right)+R_{i 3} b_{2}\left(1-b_{1}\right)\right] A_{i}$

4. $A_{i 2}=\left[R_{i 1} b_{1}\left(1-b_{2}\right)+R_{i 2} b_{2}\left(1-b_{1}\right)+R_{i 3} b_{1} b_{2}\right] A_{i}$

5. $A_{i 3}=\left[R_{i 1} b_{2}\left(1-b_{1}\right)+R_{i 2} b_{1} b_{2}+R_{i 3} b_{1}\left(1-b_{2}\right)\right] A_{i}$

Although a thinned area with various thinning intensity levels is helpful to biodiversity, allowing too many thinned areas to be heavily thinned can result in negative effects of soil erosion or biological damage. Therefore, forest managers must reduce the ratio of the thinned area that is allocated to heavy thinning intensity level by setting the thinned area $\operatorname{ratio}\left(R_{i j}\right)$ for each thinning intensity level $\left(T_{i h}, T_{i m}, T_{i l}\right)$ as $(0.1,0.2,0.7)$. The symbol $C_{i k}(B)$ is a binary variable function that creates Eqs. 3, 4, and 5 to control the meanings of $\left(A_{i 1}, A_{i 2}, A_{i 3}\right)$. For example, the combination of $\left(b_{1}, b_{2}\right)=(1,1)$ or $(1,0)$ or $(0,1)$ forces the $A_{i 1}$ to be thinned heavily with $0.1^{*} A_{i}$ areas, or at a medium thinning intensity level with $0.2 * A_{i}$ areas, or a low thinning intensity level with $0.7^{*} A_{i}$ area.

After the introduction of multi-coefficient GP, the framework of a thinning schedule is designed as follows. Firstly, two horizons covering 20 years are established, because each Forestry District in Taiwan must propose a forestry management plan every 10 years. Secondly, only one thinning entry time is defined for each 5-year period, and the thinned area is not thinned again to avoid soil erosion in fragile forestlands. Thirdly, there are five thinning prescriptions to be practiced on the forest: thinning during the first period, thinning during the second period, thinning during the third period, thinning during the fourth period, and no thinning. The thinning intensities remove 20,40 , and $60 \%$ of the volume from the thinned forest. Fourthly, the forest structure and carbon sequestration is improved by assigning a thinned area for each thinning intensity level. Fine-tuning of the thinned area is not possible using current GP, but is with multi-coefficient GP.

\subsection{Model formulation of this study}

The models that are formulated in this study use one goal and three constraints. Since the relationship between biodiversity and thinning cannot be quantified, maximizing carbon sequestration is the only goal that can be formulated using LP in the equation. Biodiversity is achieved through the edge effect that is generated by the differently thinned areas allocated using the multicoefficient GP. One of the three constraints is the restriction of the thinned area in each period, a second is the amount of wood from thinning after four periods, and the third is the even-flow control of thinning wood. The equations for the goal and constraints are shown below and detailed in the following section.

$$
\begin{aligned}
\operatorname{Max} C O_{2}= & \sum_{i=1}^{4} \sum_{j=1}^{5} \sum_{k=1}^{3}\left(S_{i j k} B_{i j k}(B) G_{i j k}\right) \\
& \times 0.319 \times 0.4974 \times 44 / 12
\end{aligned}
$$

$S_{i j k}=V_{i j k}-w_{i j k}$

$V_{i j k}=\left(1+B_{(i-1) j k}(B) G_{(i-1) j k}\right) \times S_{(i-1) j k}$

$\sum_{k=1}^{3} x_{i j k} \leq X / 4$

$\sum_{i=1}^{4} \sum_{j=1}^{5} \sum_{k=1}^{3} w_{i j k} \leq V^{\text {initial }} \times 2 \% \times 4 \times 5$

$0.9 \times \sum_{j=1}^{4} \sum_{k=1}^{3} w_{(i-1) j k} \leq \sum_{j=1}^{4} \sum_{k=1}^{3} w_{i j k} \leq 1.1 \times \sum_{j=1}^{4} \sum_{k=1}^{3} w_{(i-1) j k}$

$w_{i j k}=v_{i j k} B_{i j k}(B) T_{i j k} x_{i j k}$

$\sum_{k=1}^{3} B_{i j k}(B) x_{i j k}=B_{i j k}(B) R_{i j k} x_{i}$ 


\subsection{Three constraints used in this study}

The area constraint in Eq. 4 is the first constraint that prevents the sum of the thinned areas in each period from exceeding a quarter of the total area $(X)$. The variable, $x_{i j k}$, represents the thinned area in the $i$ th period $(i=1,2,3$, $4)$. The five thinning options are expressed as: $j(j=1$ is thinning during the first period, $j=2$ is thinning during the second period, $j=3$ is thinning during the third period, $j=4$ is thinning during the fourth period, and $j=5$ is no thinning). The symbol, $k$, refers to the 20,40 , and $60 \%$ thinning intensity levels.

Equations 5 and 6 are the second and third constraints that, respectively, represent the amount of thinning wood after four periods and the even-flow control of thinning wood. Because the average growth rate for Taiwanese forest is about $2 \%$, Eq. 5 limits the thinning wood every year to less than $2 \%$ of the initial forest volume $\left(V^{\text {initial }}\right)$. The even-flow concept in each period is regulated by Eq. 6, which is a maximum of $10 \%$ more or less than the previous period's amount, to ensure stable incomes for forestry managers. The thinning wood $\left(w_{i j k}\right)$ in Eqs. 5 and 6 is calculated using the current volume per hectare $\left(v_{i j k}\right)$ and the thinned area $\left(x_{i j k}\right)$, as shown in Eq. 7. However, the amount of thinning wood is controlled by the binary serial function, $B_{i j k}(B)$. This set of a multi-coefficient GP in Eq. 8 adjusts the ratio $\left(R_{i j k}\right)$ of thinned area for each thinning intensity level.

In this study, the ratio is set according to the growth rate. For example, if the growth rates in Table 2 are $0.6115,1.2555$, and 0.8528 after 20,40 , and $60 \%$ thinning in the first period, respectively, the thinned area ratios for each level of thinning intensity are $0.2,0.5$, and $0.3\left(G_{i} / \sum G_{i}\right)$.
2.5 Setting goals in this study

Equation 1 shows the only goal that maximizes the amount of carbon sequestration after a 20 -year planning horizon. The symbol, $S_{i j k}$, refers to the current stock after thinning, which is calculated using Eq. 2. This represents the deviation between the current volume $\left(V_{i j k}\right)$ and the amount of thinning wood removed $\left(w_{i j k}\right)$. The current volume $\left(V_{i j k}\right)$ is calculated using the stock in the previous period $\left(S_{(i-1) j k}\right)$ with the growth rates after different thinning intensities $\left(G_{(i-1) j k}\right)$ in Eq. 3. The growth rate $\left(G_{i j k}\right)$ is also decided by the binary serial number function, $\left(B_{i j k}(B)\right)$, which is set using a multi-coefficient GP. Finally, the sum of the increased volume $\left(S_{i j k} B_{i j k}(B) G_{i j k}\right)$ is transferred into carbon dioxide using the parameters of the conversion factor of Sugi basic density $\left(0.319 \mathrm{Mg} \mathrm{m}^{3}\right)$ (Fukuda et al. 2003), Sugi carbon stocking (0.4974) (Lin et al. 2002), and the molecular weight of $\mathrm{CO}_{2} / \mathrm{C}(44 / 12)$.

\section{Results}

In this section, carbon sequestration is first calculated using the MSGP and the multi-coefficient GP, in order to compare the results and to demonstrate the efficiency of allocating resources to more carbon sequestration. Secondly, a fixed-ratio multicoefficient GP is used to define a thinned area for each level of thinning intensity in order to determine the biodiversity.

\subsection{Thinning schedule for carbon sequestration using a multi-coefficient GP}

Table 2 lists the carbon sequestration achieved using a MSGP and a multi-coefficient GP. These results show that the multi-

Table 2 The results of thinning scheduled by MSGP and multi-coefficient GP

\begin{tabular}{|c|c|c|c|c|c|}
\hline MSGP* & First period & Second period & third period & Fourth period & Total \\
\hline Thinning intensity $60 \%$ & 0.00 & 0.00 & 0.00 & 0.00 & 0.00 \\
\hline $40 \%$ & 658.00 & 472.00 & 0.00 & 0.00 & $1,130.00$ \\
\hline $20 \%$ & 0.00 & 0.00 & 658.00 & 596.00 & $1,254.00$ \\
\hline \multirow[t]{2}{*}{ Thinning wood $\left(\mathrm{m}^{3}\right)$} & 34294.96 & 35577.45 & 32029.98 & 35177.00 & $137,079.30$ \\
\hline & Second period & Third period & Fourth period & Fifth period & Total \\
\hline Carbon sequestration (ton) & $104,381.60$ & $108,187.90$ & $103,706.70$ & $103,517.64$ & $419,793.84$ \\
\hline Multi-coefficient GP** & First period & Second period & Third period & Fourth period & Total \\
\hline Thinning intensity $60 \%$ & 0.00 & 0.00 & 0.00 & 0.00 & 0.00 \\
\hline $40 \%$ & 658.00 & 342.00 & 47.00 & 0.00 & $1,047.00$ \\
\hline $20 \%$ & 0 & 316 & 611.00 & 524.00 & $1,451.00$ \\
\hline \multirow[t]{2}{*}{ Thinning wood $\left(\mathrm{m}^{3}\right)$} & $35,571.90$ & $36,406.58$ & $33,587.67$ & $31,222.54$ & $136,788.69$ \\
\hline & Second period & Third period & Fourth period & Fifth period & Total \\
\hline Carbon sequestration (ton) & $104,381.60$ & $108,817.50$ & $104,323.50$ & $102,986.10$ & $420,508.70$ \\
\hline
\end{tabular}

*Non-thinned area of MCGP is 249.00 ha

**Non-thinned area of multi-coefficient GP is 135.00 ha 
coefficient GP creates better thinning schedules and allows greater carbon sequestration than the MSGP. The carbon sequestration achieved using the multi-coefficient GP is 420,500 tons from a 2,498 ha thinned area. However, the carbon sequestration achieved using the MSGP is 419,700 tons from a 2,384 ha thinned area. The amounts of thinned wood harvested using these two techniques are 137,000 and $136,700 \mathrm{~m}^{3}$, respectively.

Multi-coefficient GP fine-tunes the thinned area allocation following the second period of the thinning schedule. It uses a $40 \%$ thinning intensity on 342 ha and a $20 \%$ thinning intensity on 316 ha during the second period. During the third period, a $40 \%$ thinning intensity is used on 47 ha and a $20 \%$ thinning intensity on 611 ha. Only 524 ha have a $20 \%$ thinning intensity in the fourth period. This arrangement can increase carbon sequestration because the growth rate of young forests after thinning is higher. Although the carbon sequestration achieved using the multi-coefficient GP of a 2,633 ha forest is only $0.17 \%$ higher than that for the MSGP, the carbon sequestration increment would be higher in a larger forest or for a tree species with faster growth. Multi-coefficient GP also improves the biodiversity by siting thinned areas beside each other to generate multiform edge effects and to improve the forest structure. However, the automatic control of a thinning operation schedule using a multi-coefficient GP may not suit real situations, which may require more complex combinations of thinning operations to produce various habitats and to generate multiform edges. Therefore, using the fixed-ratio multi-coefficient GP, which allocates the resource according to the managers' preference, can develop a thinning schedule that is more practicable to real forest management.

3.2 Thinning scheduled using a fixed-ratio multi-coefficient GP for carbon sequestration and biodiversity

A good method that allows forest managers to allocate the area for each thinning intensity level is to fix the ratio of the thinned areas according to the growth rates after thinning. The thinned area ratios of each level of thinning intensity are set, respectively, to $0.2,0.5$, and 0.3 . Table 3 lists the thinned areas with the different thinning intensities for each period. These combinations of thinned areas generate multiform edges for each period, such as the adjacent 20 and $40 \%$ thinned areas or between the 40 and $60 \%$ thinned areas. Thinning wood production remains at $136,700 \mathrm{~m}^{3}$ and is mainly produced using 40 and $60 \%$ thinning intensities (even though no thinning takes place in 1,033 ha). Using a fixed-ratio multicoefficient GP, forestry managers continue to generate the same thinning wood production. However, the carbon sequestration amount decreases to 377,700 tons, which is $10.16 \%$ lower than the amount produced using the multi-coefficient GP thinning schedule. Clearly, there is a tradeoff between carbon sequestration and potential biodiversity benefits. Although biodiversity is difficult to quantify, the result seems to be a reasonable compromise between carbon sequestration and potential biodiversity benefits.

\section{Discussion}

The flexible setting of a single coefficient for a multicoefficient GP allows fine-tuning of the thinning intensity levels for thinned areas. This modification can result in higher carbon sequestration from earlier thinning and a combination of areas with multiple levels of thinning intensity though the carbon sequestration improvement is not very obvious in our case.

By using a fixed-ratio multi-coefficient GP, the size of a thinned area can be controlled according to the forest environment. In this study, the growth rate after thinning is used as an indicator to determine the ratio of the areas allocated for each thinning intensity level. In a real life situation, forestry managers can modify the ratio of these thinned areas. They can decrease the ratio of the thinned area with $60 \%$ thinning intensity for fragile forestland or increase the ratio of the thinned area with $60 \%$ thinning intensity for younger forests. Regardless of the thinning operations used, such as selection thinning, strip thinning, or row thinning, these thinned areas and their edges should generate greater diversity of habitats and species.

Table 3 The thinning schedule and carbon sequestration from fixed-ratio multi-coefficient GP

\begin{tabular}{llllll}
\hline & First period & Second period & Third period & Fourth period & Total \\
\hline Thinning intensity* $60 \%$ & 195 & 126 & 90 & 69 & 480 \\
$40 \%$ & 325 & 210 & 150 & 60 & 415 \\
$20 \%$ & 139 & 84 & $33,587.67$ & $31,222.54$ & 329 \\
Thinning wood $\left(\mathrm{m}^{3}\right)$ & $35,571.90$ & $36,406.58$ & Fourth period & Fifth period & $136,788.70$ \\
& Second period & Third period & $94,092.48$ & $91,064.93$ & Total \\
Carbon sequestration (tons) & $95,499.51$ & $97,123.46$ & & & $377,780.4$ \\
\hline
\end{tabular}

*Non-thinned area is 1,033 ha 
Forestry managers are putting increasing attention on multiple strategies for forest management. In this paper, the biodiversity objective is not set as a goal in the equation, but is achieved by changing the forest structure and the edge structure. Future studies might consider both carbon sequestration and biodiversity in the formulation to plan a multiple forest management strategy if the relationships between biodiversity index and length of the edges adjacent to thinned areas can be quantified.

\section{Conclusion}

The use of a multi-coefficient GP to plan a thinning schedule has two advantages. Firstly, the multi-coefficient GP can fine-tune a thinning schedule through better resource allocation, such as the modification of the thinned area which might result in better carbon sequestration. Secondly, a fixed-ratio multi-coefficient GP can efficiently diversify a uniform forest structure by adjusting the size of the thinned area according to forestry managers' preferences, such as considering real environmental factors. In conclusion, the use of a multi-coefficient GP allows practicable planning of better thinning alternatives that can potentially increase carbon sequestration and forest biodiversity.

Conflict of interest statement Potential conflicts of interest do not exist in the study.

\section{Appendix}

Table 4 Advantages of MSGP and multi-coefficient GP in scheduling thinning

\begin{tabular}{|c|c|c|c|}
\hline Requirement & Traditional GP & MSGP & Multi-coefficient GP \\
\hline Take both thinning intensity and area as decision variables & No & Yes & Yes \\
\hline Select a proper thinning intensity for a thinned area & No & Yes & Yes \\
\hline Reduce simulation time & No & Yes & Yes \\
\hline Assign thinned area for each thinning intensity level & No & No & Yes \\
\hline Effect of stimulating average forest growth & Low & Medium & High \\
\hline Effect of increasing total carbon sequestration & Low & Medium & High \\
\hline Efficiency of changing forest structure & Low & Medium & High \\
\hline Control ratio of thinned areas with various thinning intensities & No & No & Yes \\
\hline
\end{tabular}

Table 5 Comparison of formulation of MSGP and multi-coefficient GP

\begin{tabular}{|c|c|c|}
\hline Features & MSGP & Multi-coefficient GP \\
\hline \multirow[t]{4}{*}{ Formulation } & $\min \mathrm{z}=\sum_{\mathrm{i}=1}^{\mathrm{n}}\left(d_{i}^{+}+d_{i}^{-}\right)$ & $\operatorname{minz}=\sum_{i=1}^{\mathrm{n}}\left(d_{i}^{+}+d_{i}\right)$ \\
\hline & Goals and constrants : & Goals and constrants : \\
\hline & $\sum^{m} C_{i k}(B) T_{i k} A_{i}-d_{i}^{+}+d_{i}=G_{i}$ & $\sum_{j=1}^{n} \sum_{k=1}^{m} C_{i k}(B) T_{i k} A_{i j}-d_{i}^{+}+d_{i}^{-}=G_{i}$ \\
\hline & & $\sum_{j=1}^{m} C_{i k}(B) A_{i j}=C_{i k}(B) R_{i j} A_{i}$ \\
\hline \multicolumn{3}{|l|}{ A coefficient } \\
\hline $\begin{array}{l}\text { Controlled by } \\
\text { binary function }\end{array}$ & Yes & Yes \\
\hline With multi-levels & Yes & Yes \\
\hline \multicolumn{3}{|l|}{ A decision variable } \\
\hline $\begin{array}{l}\text { Controlled by } \\
\text { binary function }\end{array}$ & No & Yes \\
\hline $\begin{array}{l}\text { Separated into } \\
\text { many parts }\end{array}$ & No & Yes \\
\hline $\begin{array}{l}\text { Ratio control of its } \\
\text { composition }\end{array}$ & No & Yes \\
\hline
\end{tabular}




\section{References}

Arp PA, Lavigne DR (1982) Planning with goal programming: a case study for multiple use of forested land. For Chron 58:225-232

Bell EF (1976) Goal programming for land use planning. USDA Forest Service, Oregon

Bertomeu M, Romero C (2001) Managing forest biodiversity: a zero-one goal programming approach. Agric Syst 68:197-213

Bertomeu M, Romero C (2002) Forest management optimisation models and habitat diversity: a goal programming approach. J Oper Res Soc 53:1175-1184

Bottoms KE, Bartlett ET (1975) Resource allocation through goal programming. J Range Manag 28:442-447

Chang CT (2007) Multi-choice goal programming. Omega 35:389-396

Chang CT, Ku CY (2011) Fuzzy multi-choice goal programming for supplier selection. Int J Oper Res Inf Syst 1:28-52

Chang CT, Ho HP, Ku CY, Liao C (2011) A MCGP decision aid for homebuyer to make the best choice. Qual Quant 45:969-983

Chang CT, Chen HM, Zhuang ZY (2012) Multi-coefficients goal programming. Comput Ind Eng 62:616-623

Chang CT, Chen HM, Zhuang ZY (2013) Integrated multi-choice goal programming and multi-segment goal programming for supplier selection considering imperfect-quality and price-quantity discounts in a multiple sourcing environment. Int J Syst Sci (In press)

Charnes A, Cooper WW (1961) Management models and industrial applications of linear programming. Wiley, New York

Chen YT, Zheng C, Chang CT (2011a) 3-level MCGP: an efficient algorithm for MCGP in solving multi-forest management problems. Scand J For Res 26:457-465

Chen YT, Zheng C, Chang CT (2011b) Efficiently mapping an appropriate thinning schedule for optimum carbon sequestration: an application of multi-segment goal programming. For Ecol Manag 262: $1168-1173$

De-Steiguer JE, Liberti L, Schuler A, Hansen B (2003) Multi-criteria decision models for forestry and natural resources management: an annotated bibliography. USDA Forest Service General Technical Report NE-307

Díaz-Balteiro L, Romero C (1998) Modeling Timber harvest scheduling problems with multiple criteria: an application in Spain. For Sci 44: 47-57

Diaz-Balteiro L, Romero C (2001) Combined use of goal programming and the analytic hierarchy process in forest management. Anal Hierarchy Process Nat Res Environ Decis Mak Manag For Ecosyst 3:81-95

Díaz-Balteiro L, Romero C (2003) Forest management optimisation models when carbon captured is considered: a goal programming approach. For Ecol Manag 174:447-457
Díaz-Balteiro L, Romero C (2008) Making forestry decisions with multiple criteria: a review and an assessment. For Ecol Manag 255: 3222-3241

Field DB (1973) Goal programming for forest management. For Sci 19: $125-135$

Fukuda M, Iehara T, Matsumoto M (2003) Carbon stock estimates for sugi and hinoki forests in Japan. For Ecol Manag 184:1-16

Hotvedt JE (1983) Application of linear goal programming to forest harvest scheduling. South J Agric Econ 15:103-108

Ignizio JP (1976) Goal programming and extensions. Lexington Books, Lexington

Lee SM (1972) Goal programming for decision analysis. Auerback, Philadelphia

Lee AHI, Kang HY, Chang CT (2009) Fuzzy multiple goal programming applied to TFT-LCD supplier selection by downstream manufacturers. Expert Syst Appl 36:6318-6325

Liao CN (2009) Formulating the multi-segment goal programming. Comput Ind Eng 56:138-141

Liao CN (2011) Fuzzy analytical hierarchy process and multi-segment goal programming applied to new product segmented under price strategy. Comput Ind Eng 61:831-841

Liao CN, Kao HP (2010) Supplier selection model using Taguchi loss function, analytical hierarchy process and multi-choice goal programming. Comput Ind Eng 58:571-577

Liao CN, Kao HP (2011) An integrated fuzzy TOPSIS and MCGP approach to supplier selection in supply chain management. Expert Syst Appl 38:10803-10811

Lin YJ, Liu CL, Lin JC (2002) Measurement of specific gravity and carbon content of important timber species in Taiwan. Taiwan J For Sci 17:291-299

Mahmoud HB, Ketata R, Romdhane TB, Ahmed SB (2011) A multiobjective-optimization approach for a piloted qualitymanagement system: a comparison of two approaches for a case study. Comput Ind 62:460-466

Mendoza GA, Martins H (2006) Multi-criteria decision analysis in natural resource management: a critical review of methods and new modeling paradigms. For Ecol Manag 230:1-22

Oliveira FD, Volpi NMP, Sanquetta CR (2003) Goal programming in a planning problem. Appl Math Comput 140:165-178

Paksoy T, Chang CT (2010) Revised multi-choice goal programming for multi-period, multi-stage inventory controlled supply chain model with popup stores in guerrilla marketing. Appl Math Model 34:3586-3598

Romero C (1991) Handbook of critical issues in goal programming. Pergamon, Oxford

Tamiz M, Jones DF, Eldarzi E (1995) A review of goal programming and its application. Ann Oper Res 58:39-54

Zhang H, Constantino M, Falcäo A (2011) Modelling forest core with integer programming. Ann Oper Res 190:41-55 